\title{
Análise do Laboratório Remoto de Robótica Educacional (LERO) como Ferramenta de Ensino e Aprendizagem de Introdução à Programação
}

\author{
Crijina C. Flores ${ }^{1}$, Railane C. Santos ${ }^{2}$, Stenio L. Araújo ${ }^{3}$ \\ ${ }^{1}$ Colegiado de Sistema de Informação \\ Instituto Federal da Bahia (IFBA) - Vitória da Conquista, BA - Brasil \\ ${ }^{2}$ Faculdade Independente do Nordeste (FAINOR) \\ Vitória da Conquista, BA - Brasil \\ ${ }^{3}$ Colegiado de Ciência da Computação \\ Universidade Estadual do Sudoeste da Bahia (UESB) \\ Vitória da Conquista, BA - Brasil \\ crijinaflores@ifba.edu.br, \{raicostasantos, stenio.longo\}@gmail.com
}

\begin{abstract}
This work describes the feasibility study about the insertion of the Laboratório Remoto de Robótica Educacional (LERO) to complement the teaching and learning of programming logic. The research was carried out in two stages: in the first stage, the class was divided into groups and performed an activity using LERO; In the second stage, four students performed the same activity and evaluated the platform individually. The data obtained through questionnaires and interviews were presented in a descriptive and quantitative manner. It was found that LERO, although presenting some limitations, can act as a complementary tool in the teaching of Computer Programming.
\end{abstract}

Resumo. Este trabalho resulta do estudo de viabilidade de inserção do Laboratório Remoto de Robótica Educacional (LERO) como forma de complementar o ensino e aprendizagem de lógica de programação. A pesquisa da qual ele parte foi realizada em duas etapas: na primeira etapa, a turma foi dividida em grupos e realizou uma atividade utilizando o LERO; na segunda etapa, quatro alunos realizaram a mesma atividade e avaliaram a plataforma de modo individual. Os dados obtidos através de questionários e entrevistas foram apresentados de maneira descritiva e quantitativa. Constatou-se que o LERO, apesar de apresentar algumas limitações, pode atuar como ferramenta complementar no ensino de Programação de Computadores.

\section{Introdução}

A aula experimental, alinhada às aulas teóricas, é essencial para o aprendizado dos alunos, uma vez que proporciona a aplicação prática dos conhecimentos em equipamentos reais a fim de obter resultados verdadeiros. Nesse contexto, o uso de 
laboratórios remotos e virtuais para o ensino tem se tornado prática frequente no meio acadêmico para as mais diversas áreas como: física, matemática e programação. Esses espaços possibilitam capacitação de um número maior de alunos, redução dos custos da instituição com laboratórios presenciais, autonomia dos alunos na aprendizagem, maior flexibilidade de horários para experimentos e aquisição de dados com modelos reais (SIEVERS JUNIOR et al, 2016).

Diante das vantagens apresentadas pelo uso dos laboratórios remotos e virtuais, foi desenvolvido o Laboratório Remoto de Robótica Educacional (LERO), que se trata de um ambiente de programação visual baseado na WEB, extensível e adaptável. $O$ LERO é voltado para o ensino e aprendizagem de programação através da robótica educacional, visando diferentes faixas etárias (FLORES, 2015).

O LERO foi desenvolvido por um grupo de pesquisadores de três instituições: Universidade de Salvador (UNIFACS), Universidade Estadual do Sudoeste da Bahia (UESB) e Faculdade Independente do Nordeste (FAINOR), no entanto o laboratório de pesquisa fica localizado na UESB. Diante da necessidade de avaliar a plataforma e conhecer sua viabilidade no ensino, uma das pesquisadoras, concluinte do curso de Engenharia de Computação da FAINOR, optou por aplicar a ferramenta aos alunos da disciplina Algoritmos e Programação de Computadores I do primeiro semestre do mesmo curso.

Este trabalho objetivou avaliar e documentar os experimentos como forma de contribuir para o avanço das pesquisas com a plataforma, bem como apresentar a viabilidade da plataforma como forma de ensino-aprendizagem em instituições públicas e privadas.

\section{Referencial Teórico}

A programação de computadores é um dos pilares de diversas áreas profissionais; por isso, tem estado presente não só nos cursos de nível superior, mas também no ensino médio. No entanto, o processo de aprendizagem de conceitos iniciais de programação de computadores é considerado complexo. Segundo Bini (2009), alguns dos pontos mais destacados são a baixa capacidade em resolução de problemas aliada a equívocos na formulação de modelos mentais adequados; a falta de motivação para executar tarefas; a dificuldade para tratar abstração; e ferramentas e linguagens não adaptadas pedagogicamente.

Muitas ferramentas que auxiliam no ensino e aprendizagem têm sido implementadas como forma de estímulo aos alunos a resolverem problemas com iniciativa e criatividade. Laboratórios remotos e virtuais são exemplos de técnicas alternativas de ensino e aprendizagem de programação de computadores.

Os laboratórios remotos são desenvolvidos com o intuito de facilitar que usuários tenham acesso a ambientes de experimentação sem a necessidade de estarem fisicamente presentes. $\mathrm{O}$ alto custo para implementação e manutenção dos laboratórios presenciais e a limitação do espaço físico são motivos para criação dos laboratórios remotos (CARDOSO; TAKAHASHI., 2011).

A robótica é utilizada na indústria, para substituir o trabalho humano e garante a redução de custos e aumento de produtividade, mas também vem sendo utilizada na medicina e em tarefas simples do dia a dia das pessoas. Esse fato, segundo Pirola 
(2010), já a torna uma ciência interdisciplinar de grandes possibilidades na educação. A interdisciplinaridade promove cooperação, trabalho, diálogo entre as pessoas, entre as disciplinas e entre outras formas de conhecimento (FAZENDA apud PIROLA, 2010).

A interdisciplinaridade destacada por Pirola remete à ideia de robótica educacional, que se trata da aplicação de robôs nas mais diversas áreas da educação, tais como física, matemática e eletrônica, e tem por objetivo facilitar o ensino e a aprendizagem, integrando conhecimentos teóricos e práticos, por meio da prática pedagógica instigadora, motivadora da aprendizagem (PINTO, 2011, p. 42).

Na pesquisa da qual resulta este trabalho, utiliza-se o já referido LERO, que consiste em um ambiente que integra a programação em blocos e a robótica, por meio de um laboratório remoto disponibilizado via WEB para usuários de diversos níveis de conhecimento.

\section{Descrição do LERO}

O LERO é uma plataforma desenvolvida para auxiliar no ensino e aprendizagem de lógica de programação bem como no desenvolvimento do raciocínio lógico, por meio da integração entre estruturas de programação em bloco e robótica. O ambiente LERO foi desenvolvido com base em plataformas de software e hardware livres, a fim de permitir que os usuários criem novas extensões (blocos) para o software e novos experimentos para o hardware. Devido a esse fato, trata-se o LERO como um ambiente extensível e adaptável.

O LERO foi desenvolvido pensando na contribuição dos laboratórios remotos como forma de minimizar o custo e a complexidade de implantação de uma infraestrutura física para ambientes de robótica. A dificuldade de implantação de um laboratório é ainda maior quando se trata de instituições públicas por não dispor de recursos financeiros, por outro lado, muitas escolas públicas possuem laboratório de informática subutilizados, que podem ser utilizados para o desenvolvimentos de atividades com o laboratório remoto.

A plataforma possibilita que alunos de diversos níveis de conhecimento desenvolvam o raciocínio para programação por meio do uso de estruturas básicas de algoritmos, tais como sequência, seleção e repetição. Por ter se tratado de uma plataforma desenvolvida recentemente e ainda não utilizada por professores de Introdução à Programação, o LERO foi escolhido para ser avaliado.

O LERO conta com três ambientes de desenvolvimento: a) o LERO Criança, desenvolvido para usuários com idade entre 8 e 12 anos, possui blocos de construção mais abstratos, com funcionalidades próximas a comandos ou verbos usuais para essa faixa etária; b) O LERO Adolescente, desenvolvido para usuários na faixa etária de 13 a 17 anos, permite trabalhar princípios mais avançados de lógica de programação, estruturas de controle de fluxo, variáveis e outras funções; e c) o LERO Extremo, que é um ambiente direcionado ao público com idade acima de 18 anos, normalmente estudantes de graduação ou pós-graduação (FLORES, 2015).

O ambiente LERO Extremo (Figura 1) foi utilizado no estudo. Esse ambiente possui todas as funcionalidades presentes no LERO Criança e Adolescente; por esse motivo, permite que os usuários iniciem os estudos com estruturas básicas e blocos 
VI Congresso Brasileiro de Informática na Educação (CBIE 2017)

Anais do XXIII Workshop de Informática na Escola (WIE 2017)

abstratos e, em seguida, estendam as funcionalidades do ambiente, criando seus próprios blocos.

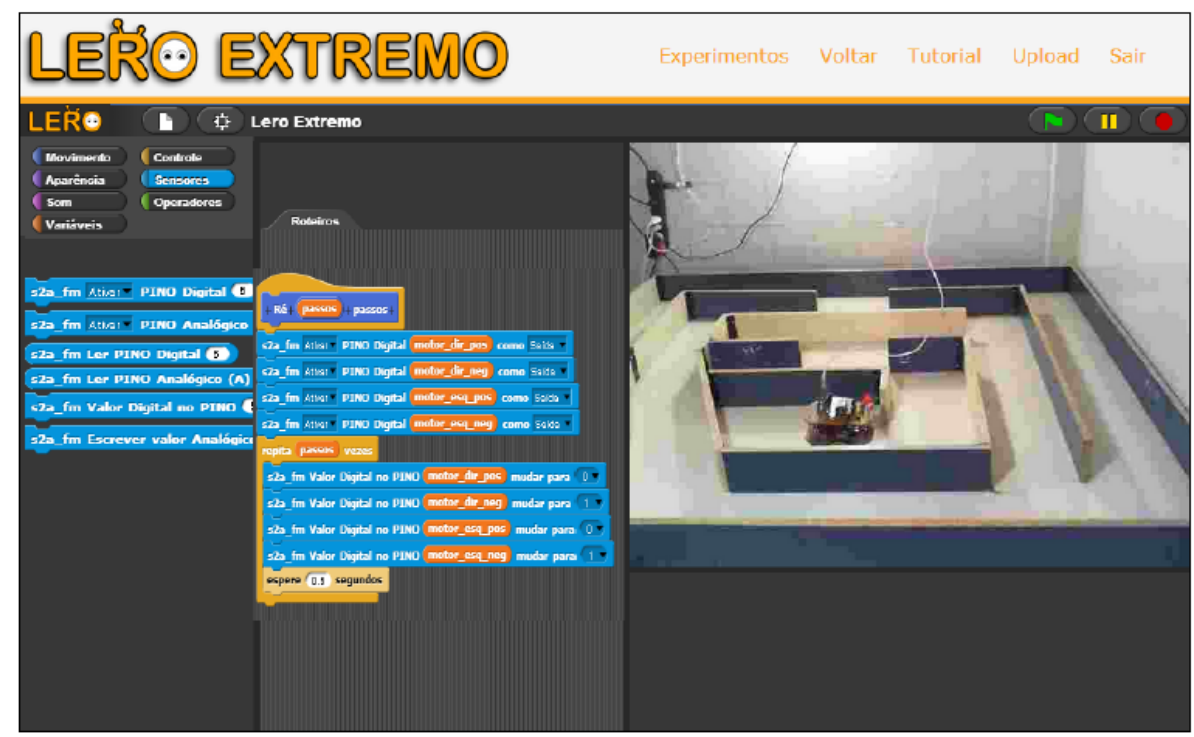

Figura 1 - Interface do LERO Extremo

\section{Metodologia Utilizada}

O experimento com a plataforma LERO foi desenvolvido para avaliar a viabilidade da inserção do uso do Laboratório Remoto de Robótica Educacional para o ensino e aprendizagem de lógica de programação.

O pré-requisito essencial foi que os alunos tivessem médio, pouco ou nenhum conhecimento em programação de computadores. $\mathrm{O}$ experimento avaliou $\mathrm{O}$ desenvolvimento dos alunos ao resolverem uma atividade proposta que exigia apenas a utilização de estruturas lógicas.

Por ser o primeiro experimento com a ferramenta, a equipe de desenvolvimento esteve presente no laboratório físico na UESB para dar suporte caso ocorressem erros. A comunicação com a equipe foi feita via celular quando necessário. Além disso, ganhouse tempo ao solicitar que, a cada tentativa, a equipe retornasse manualmente o robô ao estado inicial ao invés de fazer com que este retornasse via comandos.

Todas as atividades com o ambiente remoto foram realizadas no laboratório de informática da Fainor, cujos computadores possuem a seguinte configuração: Sistema operacional Ubuntu 12. 04 de 32 bits; memória RAM de 2GB; processador Intel Core I3 CPU 540, 3, 07 GHz x 4; HD 500 GB. Além disso, foi utilizado o navegador Google Chrome. Além disso, toda ação realizada pelos alunos na tela do computador foi capturada pelo software Kazam e salva para análises posteriores.

A pesquisa foi desenvolvida com a turma do primeiro semestre de Engenharia da Computação, em parceria com o professor da disciplina de Algoritmos e Programação de Computadores I. Na primeira unidade, o professor apresentou a todos os alunos da turma as estruturas básicas de algoritmos de programação.

Conforme o critério previamente estabelecido para realização da pesquisa, da turma de quinze alunos, três alunos que já sabiam programar participaram apenas como 
VI Congresso Brasileiro de Informática na Educação (CBIE 2017)

Anais do XXIII Workshop de Informática na Escola (WIE 2017)

espectadores do experimento.

O LERO Extremo, utilizado nesse estudo, permite que os usuários o explorem com ações mais complexas da biblioteca LERO; no entanto, exigiu-se apenas o conhecimento básico das estruturas de controle utilizadas na programação.

O estudo foi realizado em duas etapas no laboratório de informática. Na primeira etapa, os alunos foram divididos em três grupos de quatro pessoas, que foram denominados Grupo 1, Grupo 2 e Grupo 3. Além disso, houve um quarto grupo, composto por três alunos, que participaram da aula como espectadores e avaliadores da plataforma, pois não se encaixavam nos critérios pré-definidos do estudo. O trabalho em grupo foi escolhido para que os alunos pudessem discutir entre eles os processos para cumprimento do exercício, compartilhar novas ideias e conhecimento. Os componentes de cada grupo foram escolhidos via sorteio.

Antes da realização das atividades, esclareceu-se aos alunos sobre a pesquisa, seus critérios e seu objetivo; feito isso, foi-lhes entregue o Termo de Consentimento Livre e Esclarecido (TCLE) para leitura e assinatura.

$\mathrm{Na}$ Tabela 1, é apresentado o roteiro por etapas utilizado na pesquisa. A primeira etapa foi dividida em dois encontros. No primeiro encontro, foi aplicado o questionário para conhecer o perfil dos alunos em relação ao conhecimento acerca de programação de computadores e, além disso, foram fornecidas aos alunos todas as informações necessárias para a realização do experimento com o LERO Extremo.

Tabela 1 - Roteiro da pes quis a por etapas

\begin{tabular}{|c|c|c|c|c|}
\cline { 2 - 5 } & Sessão & Descrição & $\begin{array}{c}\text { Duração } \\
\text { (minutos) }\end{array}$ \\
\hline \multirow{5}{*}{ ETAPA I } & $\begin{array}{c}\text { Pré-experimento } \\
\text { (Encontro 1) }\end{array}$ & 2 & $\begin{array}{c}\text { Apresentação e contato dos } \\
\text { alunos com o ambiente. } \\
\text { Formação dos grupos. }\end{array}$ & 85 \\
\cline { 2 - 5 } & $\begin{array}{c}\text { Experimento } \\
\text { (Encontro 2) }\end{array}$ & 3 & Desenvolvimento da atividade & 100 \\
\cline { 2 - 5 } & $\begin{array}{c}\text { Pós-experimento } \\
\text { (Encontro 2) }\end{array}$ & 4 & Aplicação do questionário 2 & 30 \\
\hline \multirow{2}{*}{ ETAPA II } & (Encontro 3) & 5 & $\begin{array}{c}\text { Desenvolvimento individual } \\
\text { da atividade }\end{array}$ & 30 \\
\cline { 2 - 5 } & 6 & $\begin{array}{c}\text { Entrevista aos alunos que } \\
\text { participaram da etapa 2 }\end{array}$ & 30 \\
\hline
\end{tabular}

No segundo encontro, que aconteceu em horário de aula cedido pelo professor da disciplina, a turma realizou a atividade proposta utilizando a ferramenta e respondeu ao questionário aplicado. Na segunda etapa, a atividade foi realizada individualmente por quatro alunos; e, ao final, eles participaram de uma entrevista conduzida pela pesquisadora.

\section{Resultados e Discussão}

O projeto LERO conta com uma arena labirinto, na qual o experimento foi 
desenvolvido. Na atividade proposta, o aluno deveria utilizar as estruturas de controle (sequência, seleção e repetição), vistas em sala de aula durante a primeira unidade da disciplina, para movimentar o robô pela arena. A Figura 2(a) apresenta a arena LERO na qual o robô está localizado, e a Figura 2(b) apresenta a planta baixa da arena labirinto do LERO. O objetivo foi mover o robô do ponto A ao ponto B.

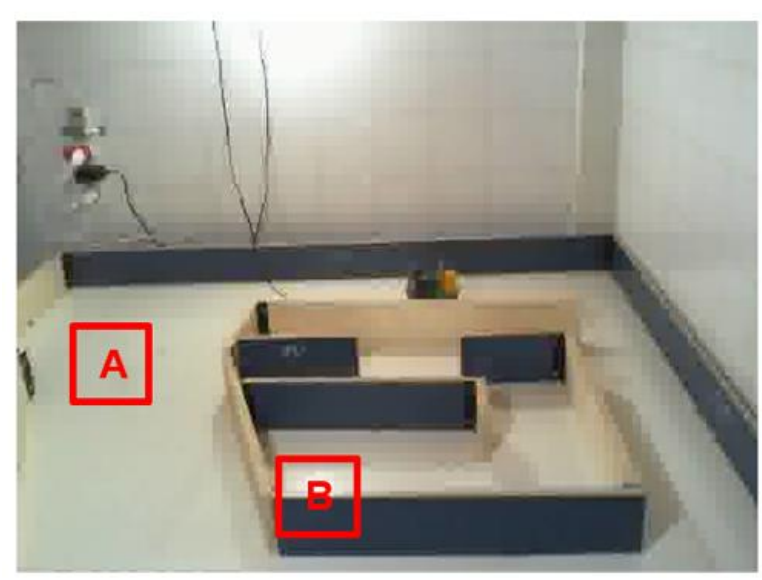

(a) Robô e Arena na Plataforma Robótica

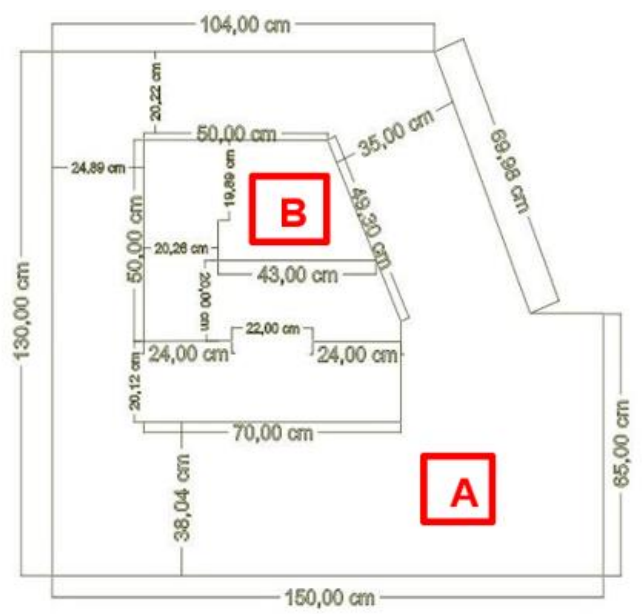

(b) Planta Baixa do Labirinto

Figura 2 - Plataforma Robótica do Ambiente LERO

Para a realização da atividade, as regras a seguir foram estabelecidas e apresentadas aos alunos:

1. O aluno deveria utilizar pelo menos uma estrutura de controle de cada tipo estudado na primeira unidade (sequência, seleção e repetição);

2. O robô não poderia tocar nas paredes da arena; as paredes foram consideradas obstáculos. Ao perceber um obstáculo, o robô deveria tomar alguma decisão (parar ou virar, por exemplo), previamente determinada pelo aluno.

No decorrer da atividade, nas duas etapas, a planta baixa da arena foi projetada no quadro para que os alunos a consultassem e desenvolvessem o roteiro com base no caminho a ser seguido e, se necessário, utilizassem a medida dos corredores e paredes do labirinto.

Durante a realização do experimento da primeira etapa, observou-se que nenhum dos grupos utilizou a estrutura de seleção. O objetivo da utilização desse tipo de comando nesse experimento era fazer com que o robô tomasse uma decisão em uma situação adversa, como no caso de desviar ao encontrar um obstáculo. Por outro lado, os três grupos utilizaram lógica sequencial e laços de repetição. A Figura 2 mostra os roteiros desenvolvidos pelos três grupos durante a atividade.

Foi observado que o Grupo 1, na Figura 3(a), utilizou conhecimentos básicos de matemática e, além disso, consultou as medidas da planta baixa da arena para calcular a quantidade de passos que o robô poderia andar até encontrar uma "entrada" para o próximo corredor. Isso aconteceu quando utilizaram os blocos "ande 5 passos" e "ande 4 passos". 


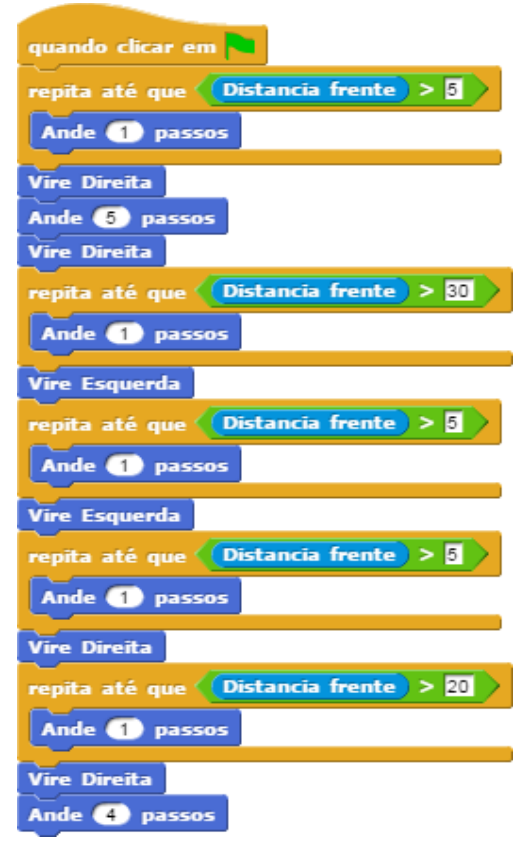

(a) Grupo1

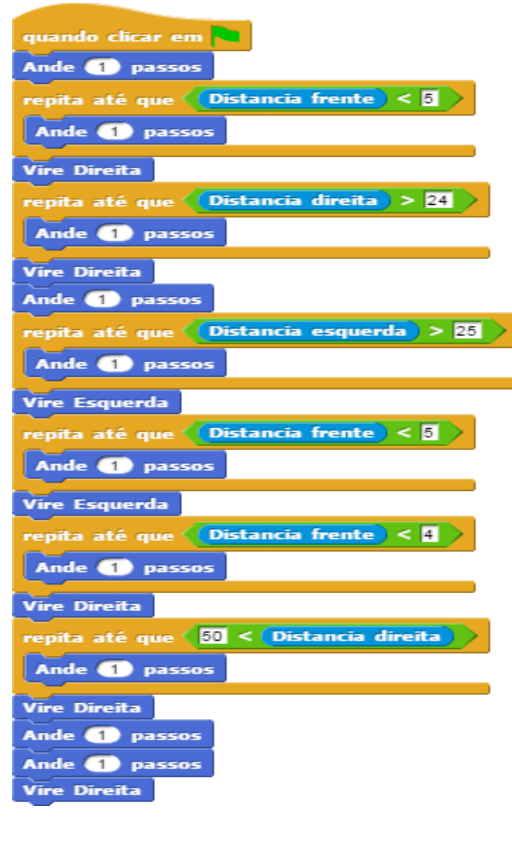

(b) Grupo 2

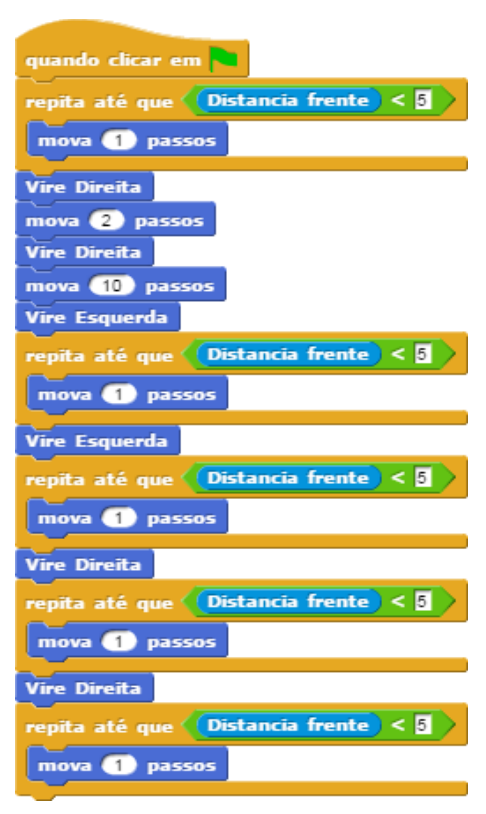

(c) Grupo 3

Figura 3. Roteiros des envolvidos pelos grupos

Os alunos também consultaram a planta para saber a que distância a porta se encontrava, pois, ao chegar nela, o robô deveria virar para adentrar no próximo corredor. Esse processo foi percebido na utilização dos laços de repetição "repita até que 'distancia frente' $>30$ " e "repita até que 'distancia frente' $>20$ ".

A Figura 3(b) mostra o roteiro elaborado pelo segundo grupo. Observa-se que, assim como o Grupo 1, este grupo não utilizou estruturas de seleção, mas utilizou laços de repetição e lógica sequencial. Além disso, fez uso de mais sensores, os da esquerda e direita.

Nesta etapa, algumas falhas na conexão da internet impediram que as sessões cumprissem plenamente o objetivo do experimento.

O roteiro desenvolvido pelo Grupo 3, mostrado na Figura 3(c), mostra que, assim como o dos grupos anteriores, também não foram utilizadas estruturas de seleção, mas uma lógica sequencial e laços de repetição. O grupo fez uso apenas dos sensores frontais e utilizou o comando "mova" ao invés do comando "ande".

Os alunos se empenharam em desenvolver um roteiro que cumprisse a missão de fazer o robô andar do estado inicial até o estado final, previamente definidos pela pesquisadora. Além disso, não houve erros de compilação, uma vez que o próprio sistema LERO indica, com uma mensagem, esse tipo de erro.

Após a realização da atividade na primeira etapa, os alunos foram submetidos a um questionário para avaliarem o uso da plataforma LERO em diversos aspectos: satisfação em utilizar a ferramenta, problemas encontrados na resolução das atividades e vantagens e desvantagens de utilizar um ambiente remoto como técnica de ensino e aprendizagem. 
VI Congresso Brasileiro de Informática na Educação (CBIE 2017)

Anais do XXIII Workshop de Informática na Escola (WIE 2017)

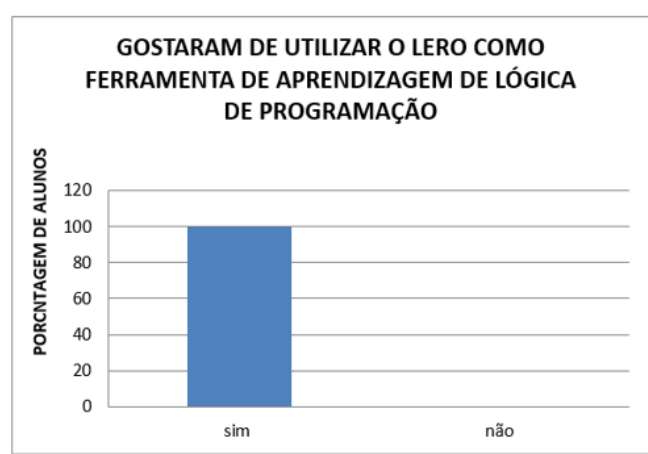

(a) Opinião dos alunos sobre o uso do LERO como ferramenta de aprendizagem de lógica de programação

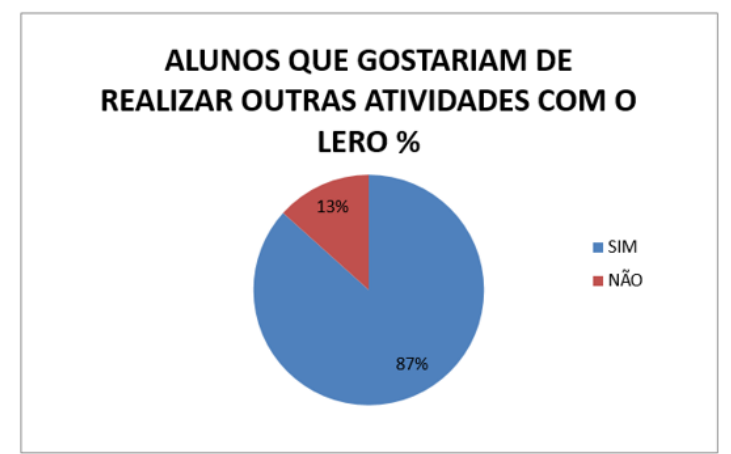

(b) Opinião dos alunos sobre realizar outras atividades com o LERO

Figura 4 - Opinião dos alunos quanto ao us o do LERO

É possível observar, na Figura 4(a), que todos os alunos gostaram de utilizar o LERO como ferramenta de aprendizagem de lógica de programação e, na Figura 4(b), que $87 \%$ dos alunos gostariam de realizar novas atividades com a plataforma.

Os dois próximos quesitos abordados no questionário aplicado estão relacionados às vantagens e desvantagens de utilizar ambientes remotos como técnica de ensino e aprendizagem de programação. Os alunos listaram diversas vantagens, tais como: aprendizado dinâmico, lúdico, atrativo; forma criativa e didática; ver o que está programando, entrar em ação e identificar os erros; uso livre da prática e em qualquer lugar, por esta ser uma ferramenta de acesso remoto; motiva o ensino. Um aluno ainda relacionou a pergunta ao uso do LERO e destacou: "Pude usar o que aprendi na sala em um robô".

Em relação às desvantagens, os alunos destacaram: não poder corrigir um erro caso ocorra, depender de uma boa conexão com internet, ter que marcar horário no site. Além disso, um aluno relatou que quem não tem base de conhecimento em programação não conseguirá utilizar o laço de repetição.

A necessidade da realização da segunda etapa da pesquisa surgiu devido à limitação com a internet. Nessa segunda etapa, quatro alunos se dispuseram a comparecer em turno oposto ao das aulas durante cinquenta minutos.

Os roteiros que os alunos desenvolveram foram semelhantes aos da primeira etapa, mas com boas condições de internet. Essa etapa foi crucial para identificar algumas limitações no LERO que impossibilitaram o sucesso no cumprimento total da atividade proposta, sendo que as principais estão relacionadas ao robô.

Durante o experimento, a equipe de desenvolvimento esteve presente no laboratório físico para dar suporte caso ocorressem erros. A comunicação com a equipe foi feita via celular quando necessário. Além disso, ganhou-se tempo ao solicitar que a equipe retornasse manualmente o robô ao estado inicial ao invés de fazer com que este retornasse via comandos. Toda ação realizada na tela do computador foi capturada pelo software Kazam e salva para análises posteriores da pesquisadora.

Os alunos que participaram da segunda etapa foram submetidos a uma entrevista que buscou coletar a opinião destes em relação à plataforma, tanto em relação à possível 
contribuição do aprendizado com a utilização do LERO quanto à identificação de limitações e sugestões para melhorias.

Os alunos apresentaram uma ótima aceitação da plataforma, relatando que este é um projeto bem elaborado e envolvente, além de desenvolver a capacidade do aluno para programação. Os participantes também relataram que a plataforma ajuda por permitir que o aluno faça várias tentativas até atingir o sucesso.

Ao ser questionado sobre a contribuição do LERO para o aprendizado, um dos alunos respondeu: "O modo como os algoritmos para a movimentação do robô são utilizados é bastante intuitivo, com um sistema "quebra-cabeça" bem desenhado. Isso ajuda o aluno a enxergar a programação não como uma sequência de linhas de texto, mas sim como peças de um quebra cabeça dinâmico." Esse ponto de vista demonstra que a plataforma cumpre com a proposta de possibilitar que, além de praticar os conteúdos, os alunos sintam-se atraídos pela ferramenta.

Em relação às limitações encontradas durante o experimento, os alunos relataram que o cabo de alimentação do robô pode atrapalhar o movimento do robô, e, como solução para esse problema, um aluno sugeriu que o robô poderia ser utilizado em área aberta, a fim de captar luz solar como fonte de energia.

Um dos alunos participantes relatou que gostaria de contribuir com inovações no projeto. Esse fato demonstra que, além de ser aceita como uma plataforma utilizada para aprender a programar, também atrai pesquisadores. Os alunos apresentaram ideias inovadoras, como a alimentação por energia solar e inserção de obstáculos.

\section{Considerações Finais}

O ensino e aprendizagem da disciplina de Introdução à Programação de Computadores apresentam muitos desafios. Os resultados encontrados neste estudo apontam que pode ser viável a utilização do Laboratório Remoto de Robótica Educacional (LERO) como forma de complementar o ensino e aprendizagem dessa disciplina, com atividades práticas e motivadoras para os alunos.

Pôde-se analisar as potencialidades da utilização do LERO, como método complementar de aprendizagem, por meio do empenho e da opinião expressada pelos alunos através de questionários e entrevistas e, além disso, identificar a necessidade dos alunos de ferramentas para a prática de conhecimento.

Os alunos, em sua maioria, tiveram dificuldades em utilizar laços de repetição; além disso, nenhum deles, em nenhuma das etapas, fez uso de estruturas de seleção. No entanto, apesar de o uso de estrutura de seleção ser solicitado como regra da atividade, o não uso não foi considerado um problema, pois as demais estruturas cumprem o objetivo solicitado na atividade. Cabe ressaltar que, considerando essa dificuldade dos alunos, a realização de atividades práticas constantes auxilia na identificação de erros e limitações no aprendizado teórico e possibilita o aperfeiçoamento do aluno no conteúdo.

Sendo assim, o Laboratório Remoto de Robótica Educacional pode atuar como ferramenta complementar no ensino de Programação de Computadores, por ser uma ferramenta capaz de atrair a atenção dos alunos, já que muitos destes relataram, durante o experimento, que, com a utilização do LERO, seria possível ver, de modo prático, os pontos onde estão cometendo erros e, além disso, gostariam de realizar novas atividades 
VI Congresso Brasileiro de Informática na Educação (CBIE 2017)

Anais do XXIII Workshop de Informática na Escola (WIE 2017)

na plataforma.

A plataforma LERO apresenta algumas limitações, mas trata-se de aspectos simples que podem ser reestruturados com facilidade, a fim de viabilizar a realização de novos estudos.

Ademais, diante de todas as suas características de ambiente extensível e adaptável, o LERO, que pode ser acessado de qualquer computador com internet, pode ser utilizado como prática de ensino-aprendizagem tanto em instituições privadas, quanto públicas. Destaca-se mais a contribuição que essa ferramenta pode oferecer às instituições públicas, pois nem todas oferecem condições para um laboratório físico para prática de robótica.

\section{Referências}

BINI, E. M.; KOSIANSKI, A.; O Ensino de Programação de Computadores em um ambiente criativo e motivador. In: ENCONTRO NACIONAL DE PESQUISA EM EDUCAÇÃO EM CIÊNCIAS, p. 2, 2009, Florianópolis. Disponível em: <http://posgrad.fae.ufmg.br/posgrad/viienpec/pdfs/675.pdf>. Acesso em: 05 mai. 2016.

CARDOSO, D. C.; TAKAHASHI, E. K. Experimentação remota em atividades de ensino formal: um estudo a partir de periódicos Qualis A. Revista Brasileira de Pesquisa em Educação em Ciências, vol. 11, n. 3, p. 187,2011. Disponível em: < http://nutec.ufu.br/experimentos/artigos/RBPEC11-3-2011.pdf>. Acesso em: 20 out. 2015.

FLORES, C. C.; Lero: Um Laboratório Remoto de Robótica Educacional Extensível e Adaptável. 2015. 137 f. Dissertação de Mestrado - Universidade Salvador - UNIFACS, Salvador, 2015.

PINTO, M. de C.; Aplicação de arquitetura pedagógica em curso de robótica educacional com hardware livre. 2011. 158f. Dissertação de Mestrado Universidade Federal do Rio de Janeiro, Rio de Janeiro, 2011.

PIROLA, N. A. (org.) Ensino de ciências e matemática, IV: temas de investigação [online]. São Paulo: Editora UNESP; São Paulo: Cultura Acadêmica, 2010. 244 p. ISBN 978-85-7983-081-5. Disponível em: < http://books.scielo.org/id/bpkng/pdf/pirola-9788579830815-11.pdf>. Acesso em: 07 jun. 2017.

SIEVERS JUNIOR, F.; GERMANO, J. S. E.; OLIVEIRA, J. M. P.; PANCCIONI, B.; MORZELLI, N. V.; MAFRA, T. WebLab um laboratório de acesso remoto controlado através da Internet. Um estudo de caso na Logística. Instituto Tecnológico de Aeronáutica, São Paulo. Disponível em: < http://www.fatecguaratingueta.edu.br/fateclog/artigos/Artigo_47.PDF>. Acesso em: 10 mar. 2016. 